\title{
Desarrollo de una acción de aprendizaje-servicio en cooperación para el desarrollo para el currículo de estudiantes de ingeniería Development of a service-learning action of cooperation for development in the curriculum of engineering students
}

\author{
Roque Calvo ${ }^{1}$, Pablo Quesada ${ }^{2}$, Maria Teresa Hernández ${ }^{1}$ \\ roque.calvo@upm.es,pablo.quesada@upm.es,mariateresa.hernandez@upm.es \\ ${ }^{1}$ Departamento de Ingeniería Mecánica, Química y \\ Diseño Industrial \\ Universidad Politécnica de Madrid \\ Madrid, España \\ ${ }^{2}$ Departamento de Ingeniería Eléctrica, Electrónica \\ Automática y Física Aplicada \\ Universidad Politécnica de Madrid \\ Madrid, España
}

\begin{abstract}
Resumen- El aprendizaje-servicio representa una tendencia formativa de importancia creciente en la educación superior. Los planes de estudios actuales surgidos del entorno de armonización de Bolonia buscan la preparación basada en la adquisición de competencias ligadas a la práctica profesional. El tercer sector (voluntariado, ONG's, etc.) es un campo de ejercicio profesional o de desarrollo personal de creciente interés. Campo de encuentro fértil para el ejercicio de las competencias técnicas con recursos limitados, la experiencia personal multicultural y el compromiso ético profesional. Se presenta el estudio de configuración y diseño de una acción formativa dirigida a estudiantes de ingeniería en la Universidad Politécnica de Madrid (UPM) para la introducción de la cooperación al desarrollo centrado en el saneamiento y el agua, enmarcado en el desarrollo de un proyecto de innovación educativa en curso. Se analizan y discuten las alternativas de contenidos mínimos, su estructura, formatos y canales de acceso. La configuración de la acción se diseña para su integración modular final con vocación de reconocimiento curricular y se propone y discuten el diseño, junto a los riesgos y oportunidades para alcanzar su efectividad.
\end{abstract}

Palabras clave: aprendizaje-servicio, cooperación al desarrollo, desarrollo curricular, competencias transversales.

\begin{abstract}
Service-learning represents a growing trend in higher education. The current curricula arising from the Bologna harmonization environment seek preparation based on the acquisition of competences linked to professional practice. The third sector (volunteering, NGOs, etc.) is a field of professional practice or personal development of growing interest. It is a fertile meeting point for the exercise of the technical competences with limited resources, the multicultural personal experience and the professional ethical commitment. The study of the configuration and design of a training action for engineering students at the Polytechnic University of Madrid (UPM) is presented for the introduction of development cooperation focused on sanitation and water, framed in the development of a project in progress of educational innovation. The alternatives of minimum contents, their structure, formats and access channels are analyzed and discussed. The configuration of the action is designed for its final modular integration with vocation of curricular recognition and the design is proposed and discussed, along with the risks and opportunities to reach its effectiveness.
\end{abstract}

Keywords: service-learning, cooperation to development, curricular development, transversal competences.

\section{INTRODUCCIÓN}

La formación es sus distintas modalidades de informal, no formal y formal forman un continuo en el aprendizaje a lo largo de la vida (European Commission, 2004). En el estadio de la formación formal superior en la universidad el reconocimiento establecido a partir del sistema europeo de transferencia de créditos (ECTS) ha supuesto la valorización de actividades que formaban al estudiante pero no estaban integradas en los planes de estudio de las diferentes titulaciones en su concepción original. Así el deporte, el asociacionismo universitario, la representación estudiantil en el seno de la universidad o diversas actividades que antes podrían ubicarse solo en la extensión universitaria, han pasado a constituir parte del currículo final en la formación de los estudios universitarios. Indudablemente como parte del nuevo paradigma de los estudios superiores, la formación para la empleabilidad, para la responsabilidad social en el ejercicio profesional y la sostenibilidad de la formación universitaria en el seno de una sociedad en constante transformación, ponen en primer plano de relevancia la actividad de voluntariado en general y concretamente la cooperación para el desarrollo. Centrado en estudios de ingeniería de la rama industrial, la contribución que desde la ingeniería se puede hacer a la ayuda al desarrollo es manifiesta en las diversas ONG's, asociaciones y grupos con actividad de voluntariado centrado en ello existentes en los países desarrollados. El interés educativo se plantea a la inversa: qué puede hacer la colaboración de ayuda al desarrollo por la formación de los estudiantes en ingeniería. La existencia de un tercer sector como actividad profesionalizada, confiere de un valor doble a las iniciativas formativas en torno a la cooperación para el desarrollo, que además de enriquecer la formación integral del estudiante, puede mejorar su empleabilidad (Atanasiu, V., Olteanu, P., 2010).

La posibilidad del acercamiento de las actividades de proyectos de cooperación al desarrollo contribuye a enriquecer la oferta formativa, pues los actuales estándares de acreditación de estudios universitarios, por ejemplo ABET, ASCE o EUR ACE, contemplan la dinámica del trabajo en proyectos de ingeniería un punto esencial para la formación 
integral en ingeniería (Accreditation Board for Engineering and Technology, 2017; Programa ACREDITA PLUS, 2015).

Las actividades en torno a la cooperación para el desarrollo ofrecen posibilidades significativas para incorporarse al curriculum formal a partir de acciones formativas correctamente diseñadas, que inicialmente se sitúen en el rango de formación informal o no formal, para llegar finalmente a su valorización dentro del curriculum ordinario de los alumnos de ingeniería. En los dos primeros estadios, informal $y$ no formal, tienen iniciativas disponibles principalmente desde el tercer sector. En el campo de formación formal y reglada se han incluido las prácticas académicas externas curriculares en la mayoría de los planes de estudio. Su relevancia y aceptación entre los estudiantes es grande (Calvo and D’Amato, 2015). Sin embargo su configuración académica en duración y ubicación dentro de los estudios le confiere un marco bien establecido y quizá no suficientemente flexible para encauzar las múltiples circunstancias de estudiantes. El objetivo de esta comunicación incluye: a) Constatar la conveniencia del acercamiento curricular de las actividades de cooperación al desarrollo a la enseñanza formal desde iniciativas más abiertas y progresivas. b) Presenta el análisis para desarrollo de una acción formativa novedosa encaminada a formación curricular para estudiantes de ingeniería de la Universidad Politécnica de Madrid.

\section{CONTEXTO}

Las virtudes formativas para el estudiante de ingeniería en proyectos de cooperación al desarrollo han sido identificadas en relación con las nuevas perspectivas en la formación integral del alumno de ingeniería que incluye aspectos como:

- Que los estudiantes experimenten todos los aspectos del proceso de ingeniería: identificación de problemas, valoración, diseño, implementación y seguimiento de resultados.

- Aprendizaje haciendo (learning-by-doing)

- Trabajo en equipo en proyectos reales de impacto social significativo.

- Aprendizaje y desarrollo personal en proyectos en entorno multicultural.

- Desarrollo en los estudiantes participantes de la conciencia ética profesional y el papel de la ingeniería en la comunidad, a partir de la formación curricular y de su participación directa en proyectos de cooperación.

Desde el punto de vista de su idoneidad formativa, se constata (Amadei, B. and Wallace, W.A., 2010; Mehta, K., \& Gorski, I., 2016) que el aprendizaje y competencias desarrolladas a través la experiencia de colaboración en proyectos de ayuda al desarrollo durante los estudios universitarios tiene repercusión extendida a lo largo de la vida profesional del egresado, con interés educativo, social y técnico. No obstante, si bien desde la perspectiva educativa la mejora en la formación integral del estudiante parece clara, la perspectiva desde el ámbito de la cooperación podría considerar tales colaboraciones puntuales un privilegio del estudiante en formación más que una contribución neta de cooperación para el desarrollo (Berg, D. R., Lee, T., \& Buchanan, E. 2016).
En el contexto de la UPM la cooperación para el desarrollo tiene apoyo institucional reconocido a través de los grupos formalmente constituidos. El grupo Sistemas de Agua y Saneamiento para el Desarrollo (el Grupo, en adelante) desarrolla actividades propias de cooperación desde 2004 en la ingeniería para la provisión de agua para consumo humano y saneamiento en diversos países en desarrollo en América Latina y África. Las actividades de voluntariado del Grupo aúnan las contribuciones de docentes en diversas disciplinas de la ingeniería y personal propio de la universidad junto a las colaboraciones de estudiantes o egresados. La formación formal con la asignatura de libre elección "Hidráulica aplicada a proyectos de desarrollo" representa una acción formativa reconocida accesible desde las diferentes titulaciones de la rama industrial impartidas en la Escuela Técnica Superior de Ingeniería y Diseño Industrial (ETSIDI) donde se ubica y ejerce principalmente su actividad el Grupo.

Inserto también en el currículo, pero de reciente impulso, es la realización de prácticas académicas curriculares (optativas) en las actividades de los proyectos en curso del grupo. Las actividades se realizan dentro del calendario lectivo, pero pueden extenderse durante el año académico completo incluyendo el periodo estival, lo que proporciona cierta flexibilidad en los periodos de realización de interés para insertar al estudiante en los trabajos de proyectos en curso cuya planificación puede no estar sincronizada con los calendarios académicos. Los planes de trabajo se ciñen a la realización de trabajos técnicos, coordinación y/o gestión de trabajos en curso, con el denominador común de obtener soluciones económicas, ingeniosas y sencillas para ser implementadas posteriormente.

Diversas actividades de difusión o información de las actividades del Grupo, su blog o series de conferencias programadas durante el curso académico, permiten el acercamiento de sus actividades a los estudiantes de ingeniería, egresados o personas interesadas en general. Son calificables de formación informal o en el caso de series de conferencias estructuradas de no formal. Centrado en los estudiantes de grado en ingeniería, las posibilidades de acercamiento a través de actividades curriculares se presentan en la etapa final de sus estudios. Si bien es interesante tener cubierta gran parte de formación básica y especializada, los tiempos de permanencia en los proyectos en curso se ven limitados, con lo que el número de potenciales candidatos y retención en la colaboración posterior en estas actividades son reducidos.

La necesidad de un acercamiento curricular (valorizado en el periodo de formación en la universidad) progresivo y flexible a las actividades de cooperación para el desarrollo en los momentos en que los alumnos puedan o deseen participar, se ha identificado como una mejora conveniente en el deseo de un desarrollo curricular para estudiantes de ingeniería.

Iniciativas desarrolladas en torno a las actividades de ONG's centradas en la ingeniería humanitaria (Amadei, B. and Wallace, W.A., 2010), muestran que la inserción curricular, a partir de cursos temáticos en las disciplinas técnicas y humanísticas con un enfoque en la cooperación al desarrollo, tiene interés directo para el currículo de los alumnos en estudios de graduado en ingeniería y valor para una formación integral e incluso para la empleabilidad en el tercer sector, de creciente importancia en los países desarrollados. De especial interés los contenidos además de técnicos deben incluir 
aspectos esenciales de la gestión de proyectos o los propios del contexto humano, geopolíticos o estratégicos (PérezFoguet, A. et al. 2005, Ortiz - Marcos, I., et al. 2013).

Más enfocados en una actividad de la cooperación, los estudios de postgrado diseñados en torno a la cooperación ya se encuentran dentro de la oferta académica en numerosas universidades. En particular en la Universidad Politécnica de Madrid junto a la Universidad Complutense de Madrid, el Máster en Estrategias y Tecnologías para el Desarrollo, de carácter inter universitario.

La acción formativa en desarrollo trata de cubrir una pequeña parcela de una necesidad no satisfecha por la oferta existente para acercar principalmente a estudiantes de ingeniería la cooperación desde el desarrollo en labores técnicas en torno al agua y saneamiento. Los objetivos que se persiguen en su desarrollo son:

- Acción introductoria de corta duración, con aspectos técnicos y humanos.

\section{- Atractiva como formación no formal}

- Apoyada en tecnologías de información que permita su acceso a distancia para grupos de interés inicial amplio.

- Ser parte de una formación más amplia, que incluiría actividades presenciales con el Grupo y con los proyectos de cooperación en marcha.3

El objetivo inicial introductorio de familiarización se dirige a grupos de personas (estudiantes o no) interesadas en conocer las problemáticas de cooperación para el desarrollo, aunque ciertos contenidos con aspectos más técnicos podrían interesar preferentemente a estudiantes de ingeniería. El título intencional inicial de la acción formativa es "Cooperación en ingeniería de agua y saneamiento para el desarrollo”.

La acción formativa inicial se debería desarrollar en el contexto de la formación no formal, donde se identifican 4 tipos de actividades posibles, a saber: métodos basados en la comunicación, basados en la actividad, métodos basados en la involucración social y los autodirigidos basados en la iniciativa personal del participante en la acción formativa.

El itinerario de integración de la acción formativa incluye los estadios:

1. Oferta abierta y online de la acción. Con vocación de formación informal o no formal dependiendo del perfil del interesado.

2. Diseño de actividades presenciales a partir de sesiones formativas estructuradas y/o conferencias que complementen la formación introductoria anterior.

\section{Abordar un pequeño trabajo en equipo.}

Los puntos 2 y 3 permitirían alcanzar para el conjunto de la formación su reconocimiento como de libre elección para alumnos de la UPM a través del sistema ECTS.

La formación informal tradicional en los entornos de cooperación de los proyectos de ingeniería parte de los conocimientos generales o específicos del participante en disciplinas clásicas y se enriquece progresivamente, auto dirigida y con su involucración social. La acción formativa en desarrollo trata de apoyarse inicialmente en la comunicación y posteriormente y de forma progresiva pedir la involucración personal del estudiante y su actividad en torno a los proyectos.

El grupo de interés y objetivo de la acción formativa se encuentra principalmente entre estudiantes de ingeniería en la rama industrial y secundariamente de otras disciplinas. Las características propias de la formación no formal (Council of Europe, 2004) incluye ser voluntaria, auto organizada y con motivación intrínseca de los participantes del grupo, además de estar centrada en el individuo en aprendizaje. Las tres primeras características que son rasgos que se estiman existentes en muchos individuos del grupo objetivo en su etapa de estudio en la universidad. Respecto a la capacidad para centrar el aprendizaje en torno al individuo, se estima que habrá que hacer un esfuerzo en los contenidos iniciales, con un equilibrio entre lo conveniente para formar y su atractivo para quien trata de introducirse en los temas de cooperación para el desarrollo.

\section{DESCRIPCIÓN}

El proceso de definición de los contenidos de la acción formativa se ve apoyado por la amplia experiencia en cooperación de los miembros del Grupo docentes, pero esto es a su vez un condicionante para tratar de racionalizar lo que es importante y lo que es secundario dentro del proceso formativo. La actuación como cooperate o gestor en la cooperación no pasa necesariamente por tener ordenados los canales de acceso a la información y aspectos esenciales de actuación para aquellos que ya están inmersos en la actividad.

Un aspecto inicial de interés inequívoco es el equilibrio que en la formación en este tema debe tener los contenidos humanísticos y los técnicos. Precisamente un signo diferencial de la acción planteada es estar dirigida a estudiantes con formación técnica previa, de tal manera que existe la posibilidad de profundizar en esos contenidos. En una serie de conferencias durante el cuatrimestre académico, se tomó como primera iniciativa la encuesta de estudiantes de ingeniería en relación a su percepción de la actividad en los proyectos técnicos de cooperación y la importancia de los aspectos humanos. Encuesta con escala de valoración tipo Likert, donde daban su opinión general. El resultado fue el esperado, en cuanto a que en la opinión mayoritaria de los estudiantes no preponderaba la importancia de la componente técnica sobre los aspectos humanos y de entorno socioeconómico en los proyectos de cooperación para el desarrollo.

El equilibrio necesario en los contenidos generales de conocimiento de entorno y los más técnicos se reforzó finalmente tras la tormenta de ideas entre miembros estables y no estables del grupo de cooperación. El propósito de la acción formativa es acercar con formación no formal el ámbito de cooperación para el desarrollo desde la ingeniería y en torno a las actividades del Grupo, pero requería introducir nociones básicas de los objetivos a largo plazo y el entorno humano y operativo en la cooperación, junto a los técnicos del entorno formativo inmediato de los estudiantes.

El siguiente paso en el desarrollo implicaba determinar el canal o canales más apropiados para la acción formativa. Hasta el momento el canal privilegiado ha sido el contacto personal entre cooperante y el estudiante interesado en la temática. En el caso del curso de libre elección centrado hidráulica para el desarrollo, su eficacia y cercanía han quedado demostradas. Sin embargo la idoneidad de los 
horarios y las limitaciones propias en la organización de la formación presencial llevan a considerar la opción no presencial como posible para la acción de formación introductoria. El deseo de alcanzar un número creciente de individuos con interés en acercarse de forma progresiva a las actividades de cooperación avocan a formación online inicialmente. Pero siendo el grupo objetivo de estudiantes propios de la UPM, las actividades presenciales serían necesarias posteriormente para alcanzar un reconocimiento de la acción formativa.

El Grupo dispone de blog propio donde efectivamente se puede volcar información, pero su interactividad es baja. Se barajaron opciones de crear una página web a partir de la cual ubicar los contenidos de forma interactiva y apoyados tanto en documentos ordinarios como en otros contenidos multimedia. La necesidad de un alojamiento, esfuerzo inicial en programación y necesidad de dar sostenibildad a la acción, aconsejaron considerar otras opciones, quizá menos ambiciosas, pero más efectivas en un entorno de recursos limitados.

La opción de acción formativa sobre plataforma educativa se consideró como una opción razonable y directa para los estudiantes propios de la UPM. La plataforma institucional es Moodle y reúne buenas características para formación organizada de grupos en formación formal. Sin embargo, la acción formativa en desarrollo se diseña inicialmente no formal, con iniciativa propia del individuo, que si bien puede ser estudiante matriculado en la UPM, también puede ser un egresado o algún otro interesado sin vinculación directa. Por ello y unido a las anteriores consideraciones, el abanico de posibilidades de fue dirigiendo finalmente a la formación online a través de plataformas para cursos abiertos.

\section{RESUltados}

La elección de contenidos para una acción formativa puede ser afrontada desde muchos y variados puntos de vista. En el caso de la acción que nos ocupa, se ha tratado de ir de lo general a lo particular, de manera que se pueden afrontar los contenidos sobre la cooperación de una manera global y en sus fundamentos más teóricos en primer lugar, para posteriormente detallar, ejemplificar y buscar un enfoque más práctico de los temas más concretos que son de interés en el contexto de las actividades del grupo y que interesa tratar en más profundidad.

Se elige como punto de partida los Objetivos de Desarrollo del Mileno (Naciones Unidas, 2015) que son un acercamiento a la cooperación que afronta de forma sumaria las temáticas posibles con un enfoque teórico que permite una visión práctica de los temas de manera muy inmediata.

De este modo, tras una introducción teórica a estos 17 objetivos de desarrollo, se pasa a un enfoque aplicado en el que se ejemplifican las diferentes temáticas sobre ejemplos de países concretos con su análisis particular.

Tras la introducción a la problemática se estudiarán las estructuras y organizaciones de cooperación locales, nacionales y supranacionales en los países donde el Grupo se encuentra más involucrado.

El siguiente bloque desarrolla la metodología de estudio y formulación de un proyecto real de cooperación, que será base teórica para la posible ejecución de un trabajo final posterior en equipo en el que se apliquen estos conocimientos a un ejemplo práctico.

En el siguiente gran bloque de contenidos (Figura 1) se estudiarán en concreto algunas de las tecnologías desarrolladas por el grupo de cooperación, con un enfoque principalmente técnico y dirigido a grupos de interés con bases técnicas para su seguimiento. Este módulo es susceptible de ser punto inicial de unas sesiones presenciales prácticas posteriormente, que se unirían a la realización de un trabajo en equipo en torno a los proyectos de cooperación en curso. Como resultado la acción formativa podría intentarse reconocer a nivel curricular dentro de las opciones de libre elección.

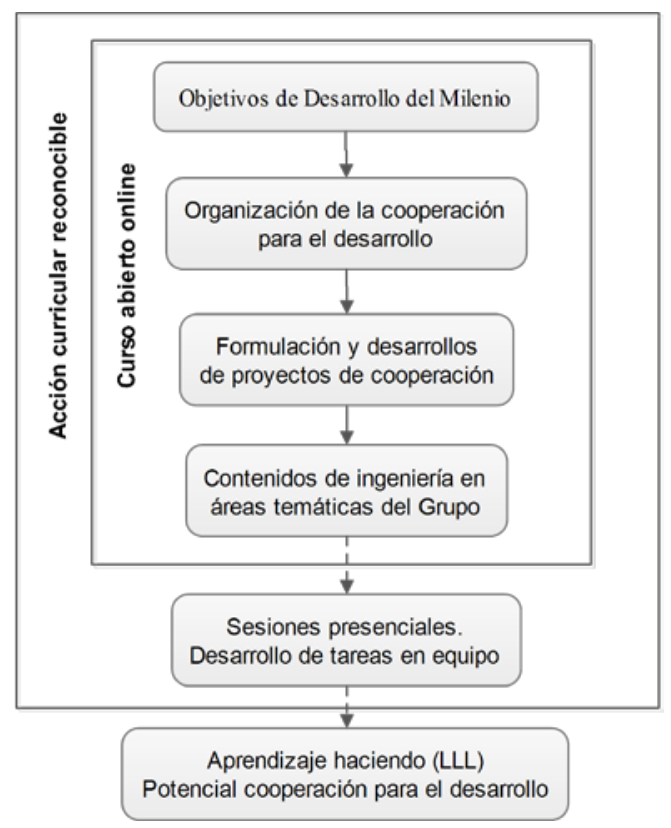

Figura 1: Diagrama de bloques de la acción formativa completa con vocación curricular.

Para desarrollar estos bloques de contenidos se han desarrollado estos módulos, dirigidos a su inclusión en la plataforma de cursos en abierto MiriadaX (Figura 2). Cada módulo estará formado por un vídeo de creación propia además de materiales y referencias externas que complementen la información y hagan la función de soporte permanente de los contenidos creados:

Al ser MiriadaX la plataforma considerada (institucional de la UPM) para el desarrollo del curso, la navegación por los distintos módulos es lineal, cada módulo se abre tras haber completado el anterior $\mathrm{y}$, al terminar el curso, todos los módulos pueden permanecer abiertos para consulta.

Justamente en la antesala del módulo inicial del curso, se realizará una encuesta general inicial, para evaluar el perfil de entrada de los alumnos y así poder valorar en mejora continua los contenidos del curso o incluso desarrollar algunos nuevos posteriormente. Al final de los módulos 1, 2, 3 y 4, se realizará una prueba de evaluación (test) de conocimientos para evaluar los conocimientos adquiridos.

Respecto de la difusión del curso, la propia elección de la plataforma MiriadaX se ha realizado debido al alcance que esta plataforma tiene en el ámbito universitario (y en especial en Latinoamérica). Además, el grupo de Sistemas de Agua y Saneamiento para el Desarrollo tiene un blog en la plataforma de blogs UPM que hace difusión de los eventos y actividades 
que organiza el grupo, con lo cual se potenciará sus mutuas posibilidades de comunicación y formación.

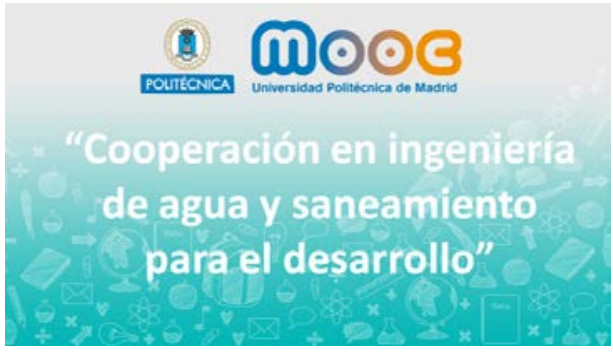

Figura 2: Maquetado inicial página inicio sobre plataforma de cursos abiertos (mooc).

El itinerario completo del esquema de formación de la Figura 1 requerirá el testeo y consolidación mínima de la acción online, para plantear sobre los resultados y el grupo de interés resultante real las acciones restantes presenciales y para los estudiantes propios de la UPM su inclusión en el currículo con la consecución efectiva del reconocimiento de los créditos académicos.

\section{CONCLUSIONES}

La mejora de la formación curricular de los alumnos de ingeniería puede beneficiarse a nivel informal y no formal del aprendizaje en torno a las actividades de los grupos de cooperación para el desarrollo en el entorno de la formación superior. La idoneidad de la formación y reforzamiento de competencias clave para el ejercicio de la ingeniería y su repercusión social, hacen de las actividades de cooperación un campo idóneo para el aprendizaje colaborativo fuera de las aulas. Bajo el esquema flexible del marco europeo de educación superior, estas actividades formativas pueden aproximarse al currículo formal. La sostenibilidad de esta iniciativa de formación se basará en los principios que sustentan la cooperación para el desarrollo, que han de ser los desencadenantes del interés de los estudiantes. La viabilidad técnica e institucional de la iniciativa se verá apoyada en su encuadre final dentro de los repositorios y plataforma institucional de la universidad junto con el reconocimiento curricular que al final del camino se espera obtener. La acción formativa intenta en última instancia incorporar las actividad de cooperación para el desarrollo al campo curricular, plenamente conscientes del valor que tanto para las competencias técnicas como humanas puede aportar a la formación de los estudiantes..

\section{AGRADECIMIENTOS}

Este trabajo se ha desarrollado como parte del Proyecto de Innovación Educativa (IE1617.5603) “Desarrollo curricular con aprendizaje-servicio y experiencial desde la cooperación de ayuda al desarrollo en la ETSIDI" financiado por la Universidad Politécnica de Madrid.

\section{REFERENCIAS}

European Commission and Council of Europe (2004). Pathways towards validation and recognition of education, training and learning in the youth field, Brussels and Strasbourg, retrieved from http://pjpeu.coe.int/documents/1017981/1668227/Pathways_towar ds_validati.pdf/caf83fd5-b4db-4b56-a1ab-

3b1178e182db último acceso1.06.2017.

Accreditation Board for Engineering and Technology, Criteria for Accrediting Engineering Programs, 2016-2017; http://www.abet.org/accreditation/accreditationcriteria/criteria-for-accrediting-engineering-programs2016-2017/, último acceso 01.06.2017.

Amadei, B., \& Sandekian, R. (2010). Model of integrating humanitarian development into engineering education. Journal of Professional Issues in Engineering Education and Practice, 136(2), 84-92.

Atanasiu Vlad, Olteanu, Paul, (2010). The revolution in education, Money Web TV in The effects of nonformal education on professional success. An analysis of student NGOs, Carmen Nicoleta Mureşan, Ulrich Teichler, International Centre for Higher Education Research Kassel, p. 13.

Berg, D. R., Lee, T., \& Buchanan, E. (2016). A methodology for exploring, documenting, and improving humanitarian service learning in the university. Journal of Humanitarian Engineering, 4(1), 10-6084.

Calvo, R., \& D’Amato, R. (2015). A collaborative method of enhancing internships evaluation through stakeholders' alignment. Procedia Engineering, 132, 167-174.

European Commission and Council of Europe (2004). Pathways towards validation and recognition of education, training and learning in the youth field, Brussels and Strasbourg, http://pjpeu.coe.int/documents/1017981/1668227/Pathways_towar ds_validati.pdf/caf83fd5-b4db-4b56-a1ab-

3b1178e182db, último acceso 01.06.2017

Mehta, K., \& Gorski, I. (2016, October). Preparing engineers for careers in social innovation and sustainable development. In Frontiers in Education Conference (FIE), 2016 IEEE (pp. 1-5).

Naciones Unidas, Objetivos de Desarrollo Sostenible (2015), http://www.undp.org/content/undp/es/home/sustainabledevelopment-goals.html, último acceso 01.06.2017.

Ortiz-Marcos, I., Cobo Benita, J. R., Aldeanueva, C. M., \& Colsa, Á. U. (2013). Competency training for managing international cooperation engineering projects. Project Management Journal, 44(2), 88-97.

Pérez-Foguet, A., Oliete-Josa, S., \& Saz-Carranza, A. (2005). Development education and engineering: A framework for incorporating reality of developing countries into engineering studies. International Journal of Sustainability in Higher Education, 6(3), 278-303.

Programa ACREDITA PLUS, Guía de evaluación para la renovación de la acreditación y la obtención del sello EUR-ACE® para títulos oficiales de Grado y de Máster en ingeniería (2015), http://eurace.iie.aneca.es/documentos/GUIA\%20ACRE DITA\%20PLUS\%20EURACE.PDF, último acceso 01.06.201 